\title{
Finite oscillator obtained through finite frame quantization
}

\author{
Nicolae Cotfas and Daniela Dragoman \\ University of Bucharest, Physics Department, \\ P.O. Box MG-11, 077125 Bucharest, Romania \\ E-mail: ncotfas@yahoo.com, danieladragoman@yahoo.com
}

\begin{abstract}
The Hamiltonian of the harmonic oscillator is usually defined as a differential operator, but an integral representation can be obtained by using the coherent state quantization. The finite frame quantization is a finite counterpart of the coherent state quantization and it allows us to define a finite oscillator by starting from the integral representation of the harmonic oscillator. Our purpose is to investigate the oscillator obtained in this way, and to present a possible application to the discrete fractional Fourier transform.
\end{abstract}

\section{Introduction}

The harmonic oscillator plays a fundamental role in quantum mechanics. A finitedimensional version, leading to Harper functions, can be obtained in a natural way by using a finite difference operator instead of the differential operator and the Fourier invariance [1]. Despite the fact that the eigenvalues and eigenfunctions can be obtained only numerically, the finite-dimensional quantum system obtained in this way has some important applications. For example, the version of the discrete fractional Fourier transform based on it 2] is used in optics and signal processing [14].

The finite frame quantization [5], which is a finite counterpart of the quantum state quantization [7], allows us to define an alternative finite-dimensional version of the harmonic oscillator [6]. Our main purpose is to investigate mathematically and numerically the quantum system with finite-dimensional Hilbert space obtained in this way, and to present an application to the discrete fractional Fourier transform.

\section{Fourier transform and the finite Fourier transform}

The Fourier transform of a function $\psi: \mathbb{R} \longrightarrow \mathbb{C}$ belonging to $L^{1}(\mathbb{R}) \cap L^{2}(\mathbb{R})$ is

$$
\mathcal{F}[\psi]: \mathbb{R} \longrightarrow \mathbb{C}, \quad \mathcal{F}[\psi](x)=\frac{1}{\sqrt{2 \pi}} \int_{-\infty}^{\infty} \mathrm{e}^{-\mathrm{i} x x^{\prime}} \psi\left(x^{\prime}\right) d x^{\prime} .
$$


This transformation can be extended to the Fourier-Plancherel transform on $L^{2}(\mathbb{R})$. It is a unitary transformation,

$$
\mathcal{F}^{-1}[\psi](x)=\frac{1}{\sqrt{2 \pi}} \int_{-\infty}^{\infty} \mathrm{e}^{\mathrm{i} x x^{\prime}} \psi\left(x^{\prime}\right) d x^{\prime}
$$

and satisfies the relations

$$
\mathcal{F}^{2}[\psi](x)=\psi(-x), \quad \mathcal{F}^{4}[\psi](x)=\psi(x), \quad \text { for any } \quad x \in \mathbb{R} .
$$

In order to obtain a finite counterpart, we consider an odd integer $d=2 s+1$ and the set

$$
\mathcal{R}_{d}=\{-s \sqrt{\delta},(-s+1) \sqrt{\delta}, \ldots,(s-1) \sqrt{\delta}, s \sqrt{\delta}\} \quad \text { with } \quad \delta=\frac{2 \pi}{d} .
$$

Each function

$$
\varphi: \mathbb{Z}_{d} \sqrt{\delta} \longrightarrow \mathbb{C}
$$

that is, each periodic function

$$
\varphi: \mathbb{Z} \sqrt{\delta} \longrightarrow \mathbb{C}
$$

satisfying the relation

$$
\varphi(n \sqrt{\delta})=\varphi(n \sqrt{\delta}+d \sqrt{\delta}), \quad \text { for any } \quad n \in \mathbb{Z}
$$

is well-determined by its restriction to the set $\mathcal{R}_{d}$. The space $l^{2}\left(\mathcal{R}_{d}\right)$ of all the functions $\varphi: \mathbb{Z}_{d} \sqrt{\delta} \longrightarrow \mathbb{C}$ considered with the scalar product

$$
\left\langle\varphi_{1}, \varphi_{2}\right\rangle=\sum_{n=-s}^{s} \overline{\varphi_{1}(n \sqrt{\delta})} \varphi_{2}(n \sqrt{\delta})
$$

is a Hilbert space isomorphic to the standard $d$-dimensional Hilbert space $\mathbb{C}^{d}$. Since

$$
\lim _{d \rightarrow \infty} \sqrt{\delta}=0 \quad \text { and } \quad \lim _{d \rightarrow \infty}( \pm s) \sqrt{\delta}= \pm \infty
$$

we can consider that, in a certain sense,

$$
\mathcal{R}_{d} \stackrel{d \rightarrow \infty}{\longrightarrow} \mathbb{R} \quad \text { and } \quad l^{2}\left(\mathcal{R}_{d}\right) \stackrel{d \rightarrow \infty}{\longrightarrow} L^{2}(\mathbb{R}) .
$$

The finite Fourier transform of a function $\varphi: \mathbb{Z} \sqrt{\delta} \longrightarrow \mathbb{C}$ from $l^{2}\left(\mathcal{R}_{d}\right)$ is the function

$$
\mathbf{F}[\varphi]: \mathbb{Z} \sqrt{\delta} \longrightarrow \mathbb{C}, \quad \mathbf{F}[\varphi](u)=\frac{1}{\sqrt{d}} \sum_{v \in \mathcal{R}_{d}} \mathrm{e}^{-\mathrm{i} u v} \varphi(v)
$$

that is, $\mathbf{F}$ is the transformation $l^{2}\left(\mathcal{R}_{d}\right) \longrightarrow l^{2}\left(\mathcal{R}_{d}\right): \varphi \mapsto \mathbf{F}[\varphi]$, where

$$
\mathbf{F}[\varphi](n \sqrt{\delta})=\frac{1}{\sqrt{d}} \sum_{k=-s}^{s} \mathrm{e}^{-\frac{2 \pi \mathrm{i}}{d} n k} \varphi(k \sqrt{\delta}) .
$$

The inverse of $\mathbf{F}$ is the adjoint transformation $l^{2}\left(\mathcal{R}_{d}\right) \rightarrow l^{2}\left(\mathcal{R}_{d}\right): \varphi \mapsto \mathbf{F}^{+}[\varphi]$, defined by

$$
\mathbf{F}^{+}[\varphi](n \sqrt{\delta})=\frac{1}{\sqrt{d}} \sum_{k=-s}^{s} \mathrm{e}^{\frac{2 \pi \mathrm{i}}{d} n k} \varphi(k \sqrt{\delta}) .
$$

This means that $\mathbf{F}^{+} \mathbf{F}=\mathbf{F} \mathbf{F}^{+}=\mathbf{I}$, where $\mathbf{I}$ is the identity operator $\mathbf{I} \varphi=\varphi$. The finite Fourier transform satisfies the relations

$$
\mathbf{F}^{2}[\varphi](u)=\varphi(-u), \quad \mathbf{F}^{4}[\varphi](u)=\varphi(u) .
$$


For $d>4$, the eigenvalues of $\mathbf{F}$ are $1,-\mathbf{i},-1$, i. The operators

$$
\begin{array}{ll}
\pi_{0}=\frac{1}{4}\left(\mathbf{I}+\mathbf{F}+\mathbf{F}^{2}+\mathbf{F}^{3}\right) & \pi_{2}=\frac{1}{4}\left(\mathbf{I}-\mathbf{F}+\mathbf{F}^{2}-\mathbf{F}^{3}\right) \\
\pi_{1}=\frac{1}{4}\left(\mathbf{I}+\mathrm{i} \mathbf{F}-\mathbf{F}^{2}-\mathrm{i} \mathbf{F}^{3}\right) & \pi_{3}=\frac{1}{4}\left(\mathbf{I}-\mathrm{i} \mathbf{F}-\mathbf{F}^{2}+\mathrm{i} \mathbf{F}^{3}\right)
\end{array}
$$

are the corresponding orthogonal projectors, and $\mathbf{F}$ admits the spectral representation

$$
\mathbf{F}=\pi_{0}-\mathrm{i} \pi_{1}-\pi_{2}+\mathrm{i} \pi_{3}=\sum_{m=0}^{3}(-\mathrm{i})^{m} \pi_{m}=\sum_{m=0}^{3} \mathrm{e}^{-\frac{\pi \mathrm{i}}{2} m} \pi_{m} .
$$

The relation

$$
\sum_{a=-s}^{s} \mathrm{e}^{\frac{2 \pi \mathrm{i}}{d} a x}=\mathrm{e}^{-\frac{2 \pi \mathrm{i}}{d} s x} \frac{\mathrm{e}^{2 \pi \mathrm{i} x}-1}{\mathrm{e}^{\frac{2 \pi \mathrm{i}}{d} x}-1} \quad \text { for any } \quad x \in \mathbb{R}-d \mathbb{Z}
$$

allows us to obtain the equality

$$
\sum_{a=-s}^{s} \mathrm{e}^{\frac{2 \pi \mathrm{i}}{d} a n}=\left\{\begin{array}{lll}
d & \text { for } & n \in d \mathbb{Z} \\
0 & \text { for } & n \notin d \mathbb{Z}
\end{array}\right.
$$

and to compute the finite Fourier transform of the coordinate function

$$
\mathfrak{q}: \mathcal{R}_{d} \longrightarrow \mathbb{R}, \quad \mathfrak{q}(\alpha)=\alpha
$$

and of its square

$$
\begin{aligned}
& \mathbf{F}[\mathfrak{q}](n \sqrt{\delta})=\frac{\sqrt{2 \pi}}{d} \sum_{a=-s}^{s} a \mathrm{e}^{-\frac{2 \pi \mathrm{i}}{d} a n}=\left\{\begin{array}{cc}
0 & \text { for } n \in d \mathbb{Z} \\
(-1)^{n} \frac{\mathrm{i} \sqrt{\pi}}{\sqrt{2} \sin \frac{\pi}{d} n} & \text { for } n \notin d \mathbb{Z}
\end{array}\right. \\
& \mathbf{F}\left[\mathfrak{q}^{2}\right](n \sqrt{\delta})=\frac{2 \pi}{d \sqrt{d}} \sum_{a=-s}^{s} a^{2} \mathrm{e}^{-\frac{2 \pi \mathrm{i}}{d} a n}=\left\{\begin{array}{cc}
\frac{2 \pi}{\sqrt{d}} \frac{s(s+1)}{3} & \text { for } n \in d \mathbb{Z} \\
(-1)^{n} \frac{\pi \cos \frac{\pi}{d} n}{\sqrt{d} \sin ^{2} \frac{\pi}{d} n} & \text { for } n \notin d \mathbb{Z}
\end{array}\right.
\end{aligned}
$$

\section{Heisenberg-Weyl group and a finite counterpart}

A quantum-mechanical system with one degree of freedom can be described by using the coordinate operator $\hat{q}$ and the momentum operator $\hat{p}$. The set

$$
\left\{\mathrm{e}^{\mathrm{i} t} \mathcal{D}(\alpha, \beta) \mid t, \alpha, \beta \in \mathbb{R}\right\} \quad \text { where } \quad \mathcal{D}(\alpha, \beta)=\mathrm{e}^{-\frac{\mathrm{i}}{2} \alpha \beta} \mathrm{e}^{i \beta \hat{q}} \mathrm{e}^{-i \alpha \hat{p}}
$$

considered with the multiplication law defined by

$$
\mathcal{D}\left(\alpha_{1}, \beta_{1}\right) \mathcal{D}\left(\alpha_{2}, \beta_{2}\right)=\mathrm{e}^{-\frac{i}{2}\left(\alpha_{1} \beta_{2}-\alpha_{2} \beta_{1}\right)} \mathcal{D}\left(\alpha_{1}+\alpha_{2}, \beta_{1}+\beta_{2}\right) .
$$

is a group, called the Heisenberg-Weyl group. In the coordinate representation [15]

$$
\begin{aligned}
& \hat{q} \psi(q)=q \psi(q), \\
& \hat{p}=-\mathrm{i} \frac{d}{d q} \\
& \mathcal{D}(\alpha, \beta) \psi(q)=\mathrm{e}^{-\frac{i}{2} \alpha \beta} \mathrm{e}^{i \beta q} \psi(q-\alpha) .
\end{aligned}
$$

The operator $\hat{p}$ satisfies the relation $\hat{p}=\mathcal{F}^{+} \hat{q} \mathcal{F}$ leading to the integral representation

$$
(\hat{p} \psi)(q)=\frac{1}{2 \pi} \int_{\mathbb{R}^{2}} x \mathrm{e}^{\mathrm{i} x y} \psi(q-y) d x d y .
$$


The linear operator $\mathrm{Q}: l^{2}\left(\mathcal{R}_{d}\right) \longrightarrow l^{2}\left(\mathcal{R}_{d}\right)$ defined by the relation

$$
\mathrm{Q} \varphi(u)=u \varphi(u) \quad \text { for } \quad u \in \mathcal{R}_{d}
$$

can be regarded as a finite counterpart of $\hat{q}$. The set $\left\{\varepsilon_{n}\right\}_{n=-s}^{s} \subset l^{2}\left(\mathcal{R}_{d}\right)$, where

$$
\varepsilon_{n}(k \sqrt{\delta})=\delta_{n k} \quad \text { for } \quad k \in\{-s,-s+1, \ldots, s-1, s\}
$$

is an orthonormal basis, and by using Dirac's notation, we have

$$
\mathbf{Q}=\sum_{n=-s}^{s} n \sqrt{\delta}\left|\varepsilon_{n}\right\rangle\left\langle\varepsilon_{n}\right| \quad \text { and } \quad \mathbf{F}=\frac{1}{\sqrt{d}} \sum_{n, m=-s}^{s} \mathrm{e}^{-\frac{2 \pi \mathrm{i}}{d} n m}\left|\varepsilon_{n}\right\rangle\left\langle\varepsilon_{m}\right| \text {. }
$$

The conjugate momentum defined as [10, 17, 18, 19, 21, 24]

$$
\mathbf{P}: l^{2}\left(\mathcal{R}_{d}\right) \longrightarrow l^{2}\left(\mathcal{R}_{d}\right), \quad \mathbf{P}=\mathbf{F}^{+} \mathbf{Q F}
$$

satisfies the relations

$$
\begin{aligned}
& (\mathbf{P} \varphi)(u)=\frac{1}{d} \sum_{\alpha, v \in \mathcal{R}_{d}} \alpha \mathrm{e}^{\mathrm{i} \alpha v} \varphi(u-v) \\
& \left(\mathrm{e}^{-i \alpha \mathbf{P}} \varphi\right)(u)=\varphi(u-\alpha)
\end{aligned}
$$

and

$$
\mathrm{e}^{-i \sqrt{\delta} \mathbf{P}}\left|\varepsilon_{n}\right\rangle=\left|\varepsilon_{n+1}\right\rangle
$$

The finite phase space $\mathcal{R}_{d}^{2}$ is a discrete counterpart of $\mathbb{R}^{2}$ and the unitary operators

$$
\mathbf{D}(\alpha, \beta)=\mathrm{e}^{-\frac{i}{2} \alpha \beta} \mathrm{e}^{i \beta \mathbf{Q}} \mathrm{e}^{-i \alpha \mathbf{P}} \quad \text { where } \quad(\alpha, \beta) \in \mathcal{R}_{d}^{2}
$$

represent a finite counterpart for $\mathcal{D}(\alpha, \beta)$. They satisfy the relations [21]

$$
\begin{aligned}
& \mathbf{D}(\alpha, \beta) \varphi(u)=\mathrm{e}^{-\frac{\mathrm{i}}{2} \alpha \beta} \mathrm{e}^{i \beta u} \varphi(u-\alpha) \\
& \mathbf{D}\left(\alpha_{1}, \beta_{1}\right) \mathbf{D}\left(\alpha_{2}, \beta_{2}\right)=\mathrm{e}^{-\frac{\mathrm{i}}{2}\left(\alpha_{1} \beta_{2}-\alpha_{2} \beta_{1}\right)} \mathbf{D}\left(\alpha_{1}+\alpha_{2}, \beta_{1}+\beta_{2}\right)
\end{aligned}
$$

and define a projective representation of a finite version of the Heisenberg-Weyl group.

\section{Ground state of the harmonic oscillator and a finite counterpart}

Let $\kappa \in(0, \infty)$. It is well-known that the Gaussian function

$$
g_{\kappa}: \mathbb{R} \longrightarrow \mathbb{R}, \quad g_{\kappa}(x)=\mathrm{e}^{-\frac{\kappa}{2} x^{2}}
$$

satisfies the relation

$$
\frac{1}{\sqrt{2 \pi}} \int_{-\infty}^{\infty} \mathrm{e}^{-\mathrm{i} \xi x} \mathrm{e}^{-\frac{\kappa}{2} x^{2}} d x=\frac{1}{\sqrt{\kappa}} \mathrm{e}^{-\frac{1}{2 \kappa} \xi^{2}}
$$

that is,

$$
\mathcal{F}\left[g_{\kappa}\right]=\frac{1}{\sqrt{\kappa}} g_{\frac{1}{\kappa}} .
$$

The norm of $g_{1}$ is $\left\|g_{1}\right\|=\sqrt[4]{\pi}$, and the normalized function $g_{1} /\left\|g_{1}\right\|$, namely,

$$
\Psi_{0}: \mathbb{R} \longrightarrow \mathbb{R}, \quad \Psi_{0}(x)=\frac{1}{\sqrt[4]{\pi}} \mathrm{e}^{-\frac{1}{2} x^{2}}
$$


is the ground state of the harmonic oscillator in the coordinate representation.

Lemma 1. We have

$$
\sum_{\ell=-\infty}^{\infty} \mathrm{e}^{-\frac{\kappa \pi}{d}(\ell d+x)^{2}}=\frac{1}{\sqrt{\kappa d}} \sum_{\ell=-\infty}^{\infty} \mathrm{e}^{\frac{2 \pi \mathrm{i}}{d} \ell x} \mathrm{e}^{-\frac{\pi}{\kappa d} \ell^{2}} \quad \text { for any } \quad x \in \mathbb{R} .
$$

Proof. The periodic function

$$
G_{\kappa}: \mathbb{R} \longrightarrow \mathbb{R}, \quad G_{\kappa}(x)=\sum_{\alpha=-\infty}^{\infty} \mathrm{e}^{-\frac{\kappa \pi}{d}(\alpha d+x)^{2}}
$$

with period $d$ admits the Fourier expansion

$$
G_{\kappa}(x)=\sum_{\ell=-\infty}^{\infty} a_{\ell} \mathrm{e}^{\frac{2 \pi \mathrm{i}}{d} \ell x}
$$

where

$a_{\ell}=\frac{1}{d} \int_{0}^{d} \mathrm{e}^{-\frac{2 \pi \mathrm{i}}{d} \ell x} \sum_{\alpha=-\infty}^{\infty} \mathrm{e}^{-\frac{\kappa}{2}\left(\sqrt{\frac{2 \pi}{d}}(\alpha d+x)\right)^{2}} d x=\frac{1}{d} \sum_{\alpha=-\infty}^{\infty} \int_{0}^{d} \mathrm{e}^{-\frac{2 \pi \mathrm{i}}{d} \ell x} \mathrm{e}^{-\frac{\kappa}{2}\left(\sqrt{\frac{2 \pi}{d}}(\alpha d+x)\right)^{2}} d x$

By denoting $t=\sqrt{\frac{2 \pi}{d}}(\alpha d+x)$ and using (32) we get [12]

$$
\begin{aligned}
a_{\ell} & =\frac{1}{\sqrt{2 \pi d}} \sum_{\alpha=-\infty}^{\infty} \int_{\alpha \sqrt{2 \pi d}}^{(\alpha+1) \sqrt{2 \pi d}} \mathrm{e}^{-\frac{2 \pi \mathrm{i}}{d} \ell\left(t \sqrt{\frac{d}{2 \pi}}-\alpha d\right)} \mathrm{e}^{-\frac{\kappa}{2} t^{2}} d t \\
& =\frac{1}{\sqrt{2 \pi d}} \sum_{\alpha=-\infty}^{\infty} \int_{\alpha \sqrt{2 \pi d}}^{(\alpha+1) \sqrt{2 \pi d}} \mathrm{e}^{-\mathrm{i} \ell t \sqrt{\frac{2 \pi}{d}}} \mathrm{e}^{-\frac{\kappa}{2} t^{2}} d t \\
& =\frac{1}{\sqrt{2 \pi d}} \int_{-\infty}^{\infty} \mathrm{e}^{-\mathrm{i} \ell t \sqrt{\frac{2 \pi}{d}}} \mathrm{e}^{-\frac{\kappa}{2} t^{2}} d t=\frac{1}{\sqrt{\kappa d}} \mathrm{e}^{-\frac{\pi}{\kappa d} \ell^{2}}
\end{aligned}
$$

whence

$$
G_{\kappa}(x)=\frac{1}{\sqrt{\kappa d}} \sum_{\ell=-\infty}^{\infty} \mathrm{e}^{\frac{2 \pi \mathrm{i}}{d} \ell x} \mathrm{e}^{-\frac{\pi}{\kappa d} \ell^{2}}
$$

The periodic function $\mathfrak{g}_{\kappa}: \mathbb{Z} \sqrt{\delta} \longrightarrow \mathbb{R}$,

$$
\mathfrak{g}_{\kappa}(n \sqrt{\delta})=\sum_{\ell=-\infty}^{\infty} \mathrm{e}^{-\frac{\kappa \pi}{d}(\ell d+n)^{2}}=\frac{1}{\sqrt{\kappa d}} \sum_{\ell=-\infty}^{\infty} \mathrm{e}^{\frac{2 \pi \mathrm{i}}{d} \ell n} \mathrm{e}^{-\frac{\pi}{\kappa d} \ell^{2}}
$$

defined by using a Zak type transformation [23], satisfies the relation [4]

$$
\begin{aligned}
\mathfrak{g}_{\kappa}(j \sqrt{\delta}) & =G_{\kappa}(j)=\frac{1}{\sqrt{\kappa d}} \sum_{\ell=-\infty}^{\infty} \mathrm{e}^{\frac{2 \pi \mathrm{i}}{d} j \ell} \mathrm{e}^{-\frac{\pi}{\kappa d} \ell^{2}} \\
& =\frac{1}{\sqrt{\kappa d}} \sum_{n=-s}^{s} \sum_{\alpha=-\infty}^{\infty} \mathrm{e}^{\frac{2 \pi \mathrm{i}}{d} j(\alpha d+n)} \mathrm{e}^{-\frac{\pi}{\kappa d}(\alpha d+n)^{2}} \\
& =\frac{1}{\sqrt{\kappa}} \frac{1}{\sqrt{d}} \sum_{n=-s}^{s} \mathrm{e}^{\frac{2 \pi \mathrm{i}}{d} j n} \sum_{\alpha=-\infty}^{\infty} \mathrm{e}^{-\frac{\pi}{\kappa d}(\alpha d+n)^{2}}
\end{aligned}
$$

equivalent with

$$
\mathbf{F}\left[\mathfrak{g}_{\kappa}\right]=\frac{1}{\sqrt{\kappa}} \mathfrak{g}_{\frac{1}{\kappa}} .
$$

This equality has been obtained by Ruzzi [16] by using the relation

$$
\mathfrak{g}_{\kappa}(n \sqrt{\delta})=\frac{1}{\sqrt{\kappa d}} \theta_{3}\left(\frac{n}{d}, \frac{\mathrm{i}}{\kappa d}\right)
$$


and the properties of the Jacobi theta function

$$
\theta_{3}(z, \tau)=\sum_{\alpha=-\infty}^{\infty} \mathrm{e}^{\mathrm{i} \pi \tau \alpha^{2}} \mathrm{e}^{2 \pi \mathrm{i} \alpha z}
$$

Lemma 2. If the numbers $N_{r, t}$ are such that the series are absolutely convergent then

$$
\sum_{r, t=-\infty}^{\infty} N_{r, t}=\sum_{k, \ell=-\infty}^{\infty} N_{k+\ell, k-\ell}+\sum_{k, \ell=-\infty}^{\infty} N_{k+\ell+1, k-\ell .}
$$

Proof. We separate the sum as [1]

$$
\sum_{r, t=-\infty}^{\infty} N_{r, t}=\sum_{\begin{array}{c}
r, t \\
\text { both even } \\
\text { or } \\
\text { both odd }
\end{array}} N_{r, t}+\sum_{\begin{array}{c}
r, t \\
\text { one even } \\
\text { and } \\
\text { other odd }
\end{array}} N_{r, t}
$$

and use the substitutions $(r, t)=(k+\ell, k-\ell)$ and $(r, t)=(k+\ell+1, k-\ell)$.

The function $\mathfrak{g}_{1}$ is a finite counterpart of $g_{1}$ and $g_{1}^{2}=g_{2}$, but $\mathfrak{g}_{1}^{2} \neq \mathfrak{g}_{2}$.

Theorem 1. We have

$$
\mathfrak{g}_{1}^{2}(n \sqrt{\delta})=\left(2 \mathfrak{g}_{2}(0)-\mathfrak{g}_{\frac{1}{2}}(0)\right) \mathfrak{g}_{2}(n \sqrt{\delta})-\left(\mathfrak{g}_{2}(0)-\mathfrak{g}_{\frac{1}{2}}(0)\right) \mathfrak{g}_{\frac{1}{2}}(2 n \sqrt{\delta}) .
$$

Proof. By using Lemma 2 we get

$$
\begin{aligned}
\mathfrak{g}_{1}^{2}(n \sqrt{\delta}) & =\frac{1}{d} \sum_{r, t=-\infty}^{\infty} \mathrm{e}^{\frac{2 \pi \mathrm{i}}{d}(r+t) n} \mathrm{e}^{-\frac{\pi}{d} r^{2}} \mathrm{e}^{-\frac{\pi}{d} t^{2}} \\
& =\frac{1}{d} \sum_{k=-\infty}^{\infty} \mathrm{e}^{\frac{2 \pi \mathrm{i}}{d} k 2 n} \mathrm{e}^{-\frac{2 \pi}{d} k^{2}} \sum_{\ell=-\infty}^{\infty} \mathrm{e}^{-\frac{2 \pi}{d} \ell^{2}} \\
& +\frac{1}{d} \sum_{k=-\infty}^{\infty} \mathrm{e}^{\frac{2 \pi \mathrm{i}}{d}(2 k+1) n} \mathrm{e}^{-\frac{\pi}{2 d}(2 k+1)^{2}} \sum_{\ell=-\infty}^{\infty} \mathrm{e}^{-\frac{\pi}{2 d}(2 \ell+1)^{2}} \\
& =\left(2 \mathfrak{g}_{2}(0)-\mathfrak{g}_{\frac{1}{2}}(0)\right) \mathfrak{g}_{2}(n \sqrt{\delta})-\left(\mathfrak{g}_{2}(0)-\mathfrak{g}_{\frac{1}{2}}(0)\right) \mathfrak{g}_{\frac{1}{2}}(2 n \sqrt{\delta}) .
\end{aligned}
$$

With the exception of a few small values of $d$, we have $\mathfrak{g}_{2}(0) \approx 1 \approx \mathfrak{g}_{\frac{1}{2}}(0)$, and hence $\mathfrak{g}_{1}^{2} \approx \mathfrak{g}_{2}$. For example in the case $d=21$, we have $\mathfrak{g}_{2}(0) \approx 1+10^{-57}$ and $\mathfrak{g}_{\frac{1}{2}}(0) \approx 1+10^{-14}$.

The norm $\mathcal{N}=\left\|\mathfrak{g}_{1}\right\|$ of $\mathfrak{g}_{1}$ satisfies the relation

$$
\begin{aligned}
\mathcal{N}^{2} & =\frac{1}{d} \sum_{n=-s}^{s}\left(\sum_{r=-\infty}^{\infty} \mathrm{e}^{\frac{2 \pi \mathrm{i}}{d} r n} \mathrm{e}^{-\frac{\pi}{d} r^{2}}\right)^{2}=\frac{1}{d} \sum_{n=-s}^{s} \sum_{r, t=-\infty}^{\infty} \mathrm{e}^{\frac{2 \pi \mathrm{i}}{d}(r+t) n} \mathrm{e}^{-\frac{\pi}{d} r^{2}} \mathrm{e}^{-\frac{\pi}{d} t^{2}} \\
& =\frac{1}{d} \sum_{r, t=-\infty}^{\infty} \sum_{n=-s}^{s} \mathrm{e}^{\frac{2 \pi \mathrm{i}}{d}(r+t) n} \mathrm{e}^{-\frac{\pi}{d} r^{2}} \mathrm{e}^{-\frac{\pi}{d} t^{2}}=\sum_{r=-\infty}^{\infty} \mathrm{e}^{-\frac{\pi}{d} r^{2}} \sum_{\ell=-\infty}^{\infty} \mathrm{e}^{-\frac{\pi}{d}(\ell d-r)^{2}} .
\end{aligned}
$$

The finite counterpart of $\Psi_{0}$ is the periodic function $(1 / \mathcal{N}) \mathfrak{g}_{1}$, that is, the function

$$
\begin{aligned}
\mathbf{g}: \mathbb{Z} \sqrt{\delta} \longrightarrow \mathbb{R}, \quad \mathbf{g}(n \sqrt{\delta}) & =\frac{1}{\mathcal{N}} \sum_{\ell=-\infty}^{\infty} \mathrm{e}^{-\frac{\pi}{d}(\ell d+n)^{2}} \\
& =\frac{1}{\mathcal{N} \sqrt{d}} \sum_{\ell=-\infty}^{\infty} \mathrm{e}^{\frac{2 \pi \mathrm{i}}{d} \ell n} \mathrm{e}^{-\frac{\pi}{d} \ell^{2}} .
\end{aligned}
$$



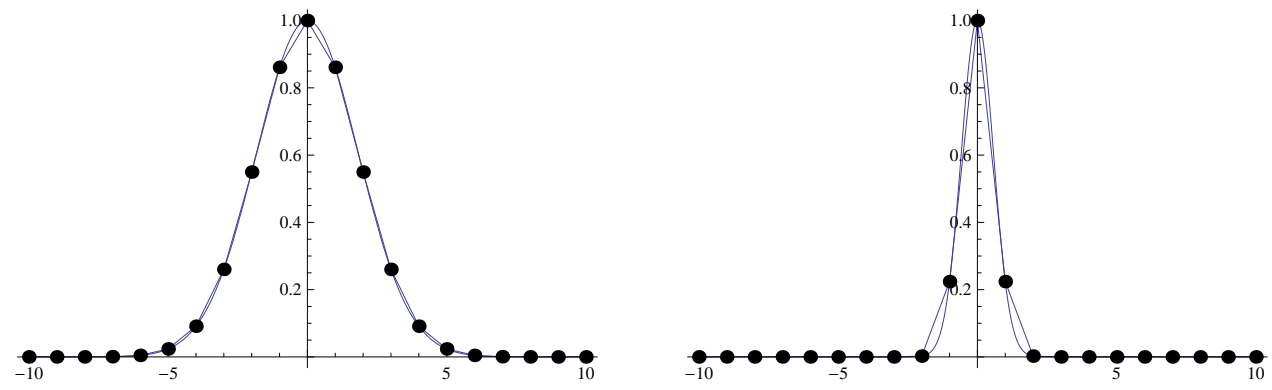

Figure 1. The functions $g_{1}, \mathfrak{g}_{1}$ (left) and $g_{10}, \mathfrak{g}_{10}$ (right) in the case $d=21$.

Theorem 2. For any $n, m \in\{-s,-s+1, \ldots, s-1, s\}$ we have

$$
\frac{1}{\sqrt{d}} \sum_{a=-s}^{s} \mathbf{g}((n-a) \sqrt{\delta}) \mathbf{g}((m-a) \sqrt{\delta})=\mathbf{F}\left[\mathbf{g}^{2}\right]((n-m) \sqrt{\delta})
$$

Proof. By using the definition of $\mathbf{g}$ we get

$$
\begin{aligned}
\sum_{a=-s}^{s} \mathbf{g}((n-a) & \sqrt{\delta}) \mathbf{g}((m-a) \sqrt{\delta})=\frac{1}{d \mathcal{N}^{2}} \sum_{r, t=-\infty}^{\infty} \sum_{a=-s}^{s} \mathrm{e}^{-\frac{2 \pi \mathrm{i}}{d} a(r+t)} \mathrm{e}^{\frac{2 \pi \mathrm{i}}{d}(r n+t m)} \mathrm{e}^{-\frac{\pi}{d} r^{2}} \mathrm{e}^{-\frac{\pi}{d} t^{2}} \\
& =\frac{1}{\mathcal{N}^{2}} \sum_{r=-\infty}^{\infty} \mathrm{e}^{\frac{2 \pi \mathrm{i}}{d} r(n-m)} \mathrm{e}^{-\frac{\pi}{d} r^{2}} \sum_{\ell=-\infty}^{\infty} \mathrm{e}^{-\frac{\pi}{d}(\ell d-r)^{2}} \\
& =\frac{1}{\mathcal{N}^{2}} \sum_{k=-s}^{s} \sum_{t=-\infty}^{\infty} \mathrm{e}^{\frac{2 \pi \mathrm{i}}{d}(t d-k)(n-m)} \mathrm{e}^{-\frac{\pi}{d}(t d-k)^{2}} \sum_{\ell=-\infty}^{\infty} \mathrm{e}^{-\frac{\pi}{d}(\ell d-t d-k)^{2}} \\
& =\frac{1}{\mathcal{N}^{2}} \sum_{k=-s}^{s} \mathrm{e}^{-\frac{2 \pi \mathrm{i}}{d} k(n-m)} \sum_{t=-\infty}^{\infty} \mathrm{e}^{-\frac{\pi}{d}(t d-k)^{2}} \sum_{\ell=-\infty}^{\infty} \mathrm{e}^{-\frac{\pi}{d}(\ell d-k)^{2}} \\
& =\sqrt{d} \mathbf{F}\left[\mathbf{g}^{2}\right]((n-m) \sqrt{\delta}) .
\end{aligned}
$$

\section{Standard coherent states and a finite counterpart}

The standard coherent states can be defined as the states

$$
|\alpha, \beta\rangle=\mathcal{D}(\alpha, \beta) \Psi_{0} \quad \text { with } \quad(\alpha, \beta) \in \mathbb{R}^{2} .
$$

They satisfy in $L^{2}(\mathbb{R})$ the resolution of the identity

$$
\mathbb{I}=\frac{1}{2 \pi} \int_{\mathbb{R}^{2}} d \alpha d \beta|\alpha, \beta\rangle\langle\alpha, \beta|
$$

that is, we have

$$
|\psi\rangle=\frac{1}{2 \pi} \int_{\mathbb{R}^{2}} d \alpha d \beta|\alpha, \beta\rangle\langle\alpha, \beta \mid \psi\rangle \quad \text { for any } \quad \psi \in L^{2}(\mathbb{R}) .
$$

For $d=2 s+1$ large enough, the norm $\mathcal{N}$ of $\mathfrak{g}_{1}$ satisfies the relation

$$
\mathcal{N}=\sqrt{\sum_{u \in \mathcal{R}_{d}}\left(\mathfrak{g}_{1}(u)\right)^{2}} \approx \sqrt[4]{\frac{d}{2}}
$$

For example, in the case $d=21$ we have (see Figure 1)

$$
\max _{u \in \mathcal{R}_{d}}\left|\mathfrak{g}_{1}(u)-g_{1}(u)\right|=1.3 \cdot 10^{-8} \quad \text { and } \quad \mathcal{N}-\sqrt[4]{\frac{d}{2}}=1.7 \cdot 10^{-14}
$$


For $d$ large enough we have

$$
\mathbf{g}(u) \approx \sqrt[4]{\delta} \Psi_{0}(u) \quad \text { for } \quad u \in \mathcal{R}_{d}
$$

The $d^{2}$ states $\left\{|\alpha, \beta\rangle_{d}\right\}_{(\alpha, \beta) \in \mathcal{R}_{d}^{2}}$, where

$$
|\alpha, \beta\rangle_{d}=\mathbf{D}(\alpha, \beta) \mathbf{g} \quad \text { with } \quad \mathbf{D}(\alpha, \beta) \mathbf{g}(u)=\mathrm{e}^{-\frac{\mathrm{i}}{2} \alpha \beta} \mathrm{e}^{\mathrm{i} \beta u} \mathbf{g}(u-\alpha)
$$

that is, the states

$$
|\alpha, \beta\rangle_{d}=\mathrm{e}^{-\frac{\mathrm{i}}{2} \alpha \beta} \sum_{n=-s}^{s} \mathrm{e}^{\mathrm{i} \sqrt{\delta} \beta n} \mathbf{g}(n \sqrt{\delta}-\alpha)\left|\varepsilon_{n}\right\rangle
$$

satisfy the resolution of the identity in $l^{2}\left(\mathcal{R}_{d}\right)$ [6, 20, 21]

$$
\mathbf{I}=\frac{1}{d} \sum_{(\alpha, \beta) \in \mathcal{R}_{d}^{2}}|\alpha, \beta\rangle_{d d}\langle\alpha, \beta| .
$$

For any $\varphi \in l^{2}\left(\mathcal{R}_{d}\right)$ we have

$$
|\varphi\rangle=\mathbf{I}|\varphi\rangle=\frac{1}{d} \sum_{(\alpha, \beta) \in \mathcal{R}_{d}^{2}}|\alpha, \beta\rangle_{d d}\langle\alpha, \beta \mid \varphi\rangle
$$

and

$$
\|\varphi\|^{2}=\left.\left.\frac{1}{d} \sum_{(\alpha, \beta) \in \mathcal{R}_{d}^{2}}\right|_{d}\langle\alpha, \beta \mid \varphi\rangle\right|^{2} .
$$

The elements of the frame [3, 9] $\left\{|\alpha, \beta\rangle_{d}\right\}_{(\alpha, \beta) \in \mathcal{R}_{d}^{2}}$, in general, are not orthogonal

$$
{ }_{d}\left\langle\alpha_{1}, \beta_{1} \mid \alpha_{2}, \beta_{2}\right\rangle_{d}=\mathrm{e}^{\frac{\mathrm{i}}{2}\left(\alpha_{1} \beta_{1}-\alpha_{2} \beta_{2}\right)} \sum_{u \in \mathcal{R}_{d}} \mathrm{e}^{\mathrm{i}\left(\beta_{2}-\beta_{1}\right) u} \mathbf{g}\left(u-\alpha_{1}\right) \mathbf{g}\left(u-\alpha_{2}\right) .
$$

The finite frame $\left\{|\alpha, \beta\rangle_{d}\right\}_{(\alpha, \beta) \in \mathcal{R}_{d}^{2}}$ is a finite counterpart of the system of standard

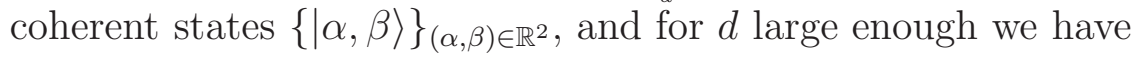

$$
\mathbf{D}(\alpha, \beta) \mathbf{g}(u) \approx \sqrt[4]{\delta} \mathcal{D}(\alpha, \beta) \Psi_{0}(u) \quad \text { for almost all } u \in \mathcal{R}_{d}
$$

The agreement is very good in the midle part of $\mathcal{R}_{d}$, but some significant differences may occur in the extreme parts (see Figure 2). In the particular case $d=21$ we have $\mathcal{R}_{21}=\{n \sqrt{\delta} \mid-10 \leq n \leq 10\}$ with $\delta=2 \pi / 21$. The values of

$$
\max _{-8 \leq n \leq 8}\left|\mathbf{D}(\alpha, \beta) \mathbf{g}(n \sqrt{\delta})-\sqrt[4]{\delta} \mathcal{D}(\alpha, \beta) \Psi_{0}(n \sqrt{\delta})\right|
$$

in certain particular cases are presented in Table 1 . In the case $(\alpha, \beta) \in \mathcal{R}_{d}^{2}$, we consider that the restriction of the function $\sqrt[4]{\delta}|\alpha, \beta\rangle$ to $\mathcal{R}_{d}$ is almost identical to $|\alpha, \beta\rangle_{d}$. As concern the Fourier transform

$$
\mathcal{F}|\alpha, \beta\rangle=|-\beta, \alpha\rangle \quad \text { and } \quad \mathbf{F}|\alpha, \beta\rangle_{d}=|-\beta, \alpha\rangle_{d} .
$$

Since $\mathbf{F}$ is a unitary operator, we have

$$
{ }_{d}\left\langle\alpha_{1}, \beta_{1} \mid \alpha_{2}, \beta_{2}\right\rangle_{d}={ }_{d}\left\langle-\beta_{1}, \alpha_{1} \mid-\beta_{2}, \alpha_{2}\right\rangle_{d} .
$$


Table 1. Differences between continuous and discrete coherent states.

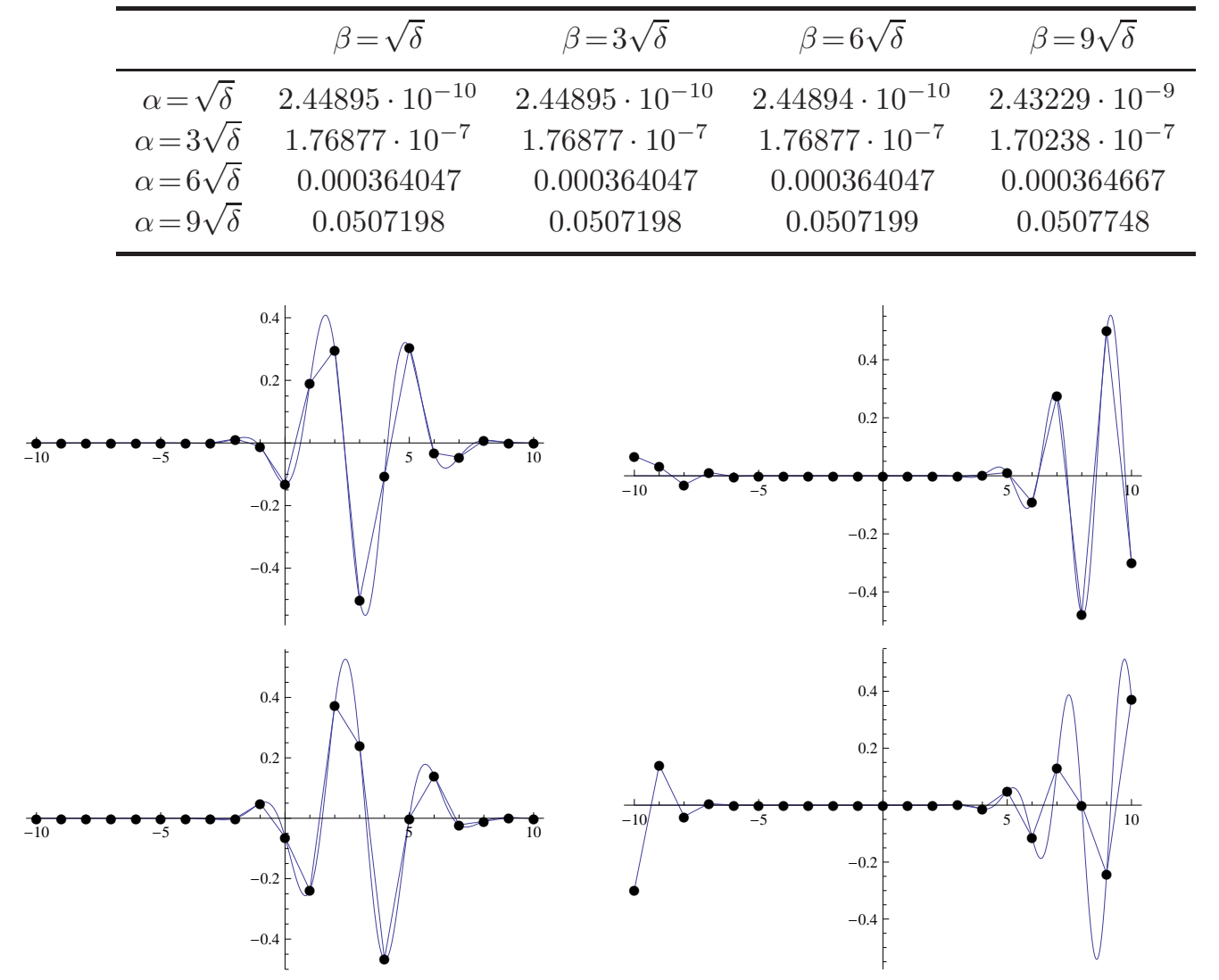

Figure 2. The real part (first line) and the imaginary part (second line) of $|3 \sqrt{\delta}, 6 \sqrt{\delta}\rangle$ versus $|3 \sqrt{\delta}, 6 \sqrt{\delta}\rangle_{21}$ (left) and $|9 \sqrt{\delta}, 9 \sqrt{\delta}\rangle$ versus $|9 \sqrt{\delta}, 9 \sqrt{\delta}\rangle_{21}$ (right).

\section{Finite oscillator obtained by using finite difference operators}

The Hamiltonian of the harmonic oscillator

$$
\hat{H}=-\frac{1}{2} \frac{\mathrm{d}^{2}}{\mathrm{~d} x^{2}}+\frac{1}{2} x^{2}=-\frac{1}{2}\left(\mathcal{D}^{2}+\mathcal{F} \mathcal{D}^{2} \mathcal{F}^{+}\right) .
$$

satisfies the relation

$$
\mathcal{F} \hat{H}=\hat{H} \mathcal{F}
$$

and the system $\left\{\Psi_{n}\right\}_{n \in \mathbb{N}}$ of Hermite-Gaussian functions

$$
\Psi_{n}: \mathbb{R} \longrightarrow \mathbb{R}, \quad \Psi_{n}(x)=\frac{1}{\sqrt{n ! 2^{n} \sqrt{\pi}}} H_{n}(x) \mathrm{e}^{-\frac{1}{2} x^{2}} .
$$

denoted sometimes by $\{|n\rangle\}_{n \in \mathbb{N}}$, is a complete othonormal set of common eigenfunctions

$$
\begin{array}{ll}
\langle n \mid k\rangle=\delta_{n k}, & \hat{H}|n\rangle=\left(n+\frac{1}{2}\right)|n\rangle, \\
\sum_{n=0}^{\infty}|n\rangle\langle n|=\mathbb{I}, & \mathcal{F}|n\rangle=(-\mathrm{i})^{n}|n\rangle .
\end{array}
$$

The finite-difference operator $\tilde{\mathcal{D}}^{2}$, where

$$
\tilde{\mathcal{D}}^{2} \varphi(x)=\frac{\varphi(x+\epsilon)-2 \varphi(x)+\varphi(x-\epsilon)}{\epsilon^{2}}
$$



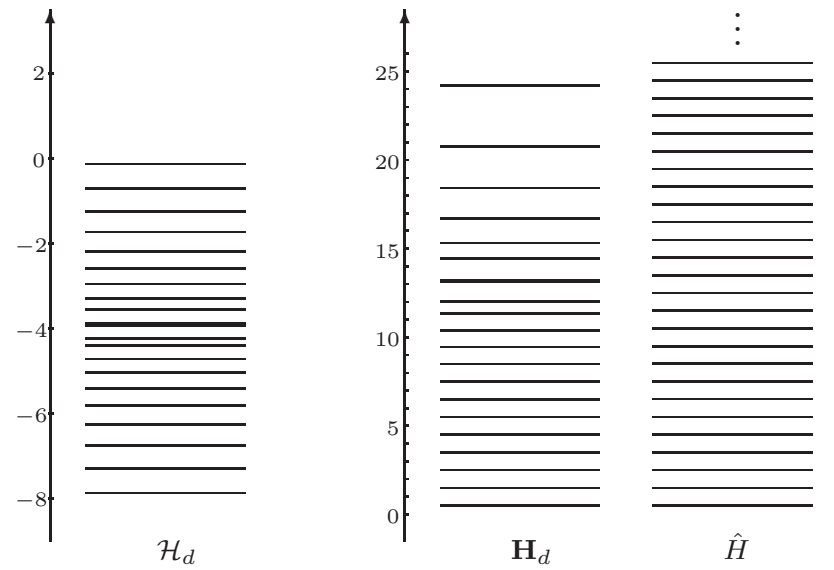

Figure 3. The eigenvalues of $\hat{H}$ and the eigenvalues of $\mathcal{H}_{d}$ and $\mathbf{H}_{d}$ in the case $d=21$.

is an approximation of $\mathcal{D}^{2}$, and for $x=n \epsilon$ with $\epsilon=\sqrt{\delta}$ we get

$$
\mathbf{F} \tilde{\mathcal{D}}^{2} \mathbf{F}^{+}=2 d\left(\cos \frac{2 \pi n}{d}-1\right) .
$$

The finite-difference Hamiltonian

$$
\mathcal{H}_{d}=\tilde{\mathcal{D}}^{2}+\mathbf{F} \tilde{\mathcal{D}}^{2} \mathbf{F}^{+}
$$

commutes with the finite Fourier transform

$$
\mathbf{F} \mathcal{H}_{d}=\mathcal{H}_{d} \mathbf{F}
$$

By denoting $\varphi[n]:=\varphi(n \sqrt{\delta})$, the equation $\mathcal{H}_{d} \varphi=\lambda \varphi$ becomes

$$
\varphi[n+1]-2 \varphi[n]+\varphi[n-1]+2\left(\cos \frac{2 \pi n}{d}-1\right) \varphi[n]=\lambda \varphi[n] .
$$

It is known [1] that the eigenvalues of $\mathcal{H}_{d}$ are distinct for odd $d$ (see Figure 3). Therefore, the eigenfunctions of $\mathcal{H}_{d}$ are at the same time eigenfunctions of $\mathbf{F}$. The normalized eigenfunctions $\mathbf{h}_{m}$ of $\mathcal{H}_{d}$, considered in the increasing order of the number of sign alternations, satisfy the relation [1, 22]

$$
\mathbf{F} \mathbf{h}_{m}=(-\mathrm{i})^{m} \mathbf{h}_{m}
$$

and are called Harper functions. They correspond to the eigenvectors of the matrix

$$
\left(2\left(\cos \frac{2 \pi n}{d}-2\right) \delta_{n m}+\delta_{n, m+1}+\delta_{n, m-1}+\delta_{n, m-2 s}+\delta_{n, m+2 s}\right)_{-s \leq n, m \leq s}
$$

and can be regarded as a finite version of Hermite-Gaussian functions $\Psi_{0}, \Psi_{1}, \ldots, \Psi_{d-1}$.

\section{Finite oscillator obtained by using the finite frame quantization}

The operator corresponding to the function

$$
f: \mathbb{R}^{2} \longrightarrow \mathbb{R}, \quad f(\alpha, \beta)=\frac{\alpha^{2}+\beta^{2}}{2}
$$

through the coherent state quantization [7], namely,

$$
A_{f}=\frac{1}{2 \pi} \int_{\mathbb{R}^{2}} d \alpha d \beta \frac{\alpha^{2}+\beta^{2}}{2}|\alpha, \beta\rangle\langle\alpha, \beta|
$$


is the Hamiltonian of a translated harmonic oscillator [7]

$$
A_{f}=\sum_{n=0}^{\infty}(n+1)|n\rangle\langle n|=-\frac{1}{2} \frac{\mathrm{d}^{2}}{\mathrm{~d} x^{2}}+\frac{1}{2} x^{2}+\frac{1}{2}=\hat{H}+\frac{1}{2}
$$

The relation

$$
\hat{H}=-\frac{1}{2}+\frac{1}{2 \pi} \int_{\mathbb{R}^{2}} d \alpha d \beta \frac{\alpha^{2}+\beta^{2}}{2}|\alpha, \beta\rangle\langle\alpha, \beta|
$$

can be regarded as an integral representation of the harmonic oscillator Hamiltonian.

The linear operator corresponding to the function

$$
\mathbf{f}: \mathcal{R}_{d}^{2} \longrightarrow \mathbb{R}, \quad \mathbf{f}(\alpha, \beta)=\frac{\alpha^{2}+\beta^{2}}{2}
$$

through the finite frame quantization, namely,

$$
\mathbf{A}_{\mathbf{f}}: l^{2}\left(\mathcal{R}_{d}\right) \longrightarrow l^{2}\left(\mathcal{R}_{d}\right), \quad \mathbf{A}_{\mathbf{f}}=\frac{1}{d} \sum_{(\alpha, \beta) \in \mathcal{R}_{d}^{2}} \frac{\alpha^{2}+\beta^{2}}{2}|\alpha, \beta\rangle_{d d}\langle\alpha, \beta| .
$$

can be regarded [6] as a finite counterpart of $A_{f}$. The operator

$$
\mathbf{H}_{d}=\mathbf{A}_{\mathbf{f}}-\frac{1}{2}
$$

is the Hamiltonian of a finite oscillator. We prove that, in a certain sense,

$$
\hat{H}=\lim _{d \rightarrow \infty} \mathbf{H}_{d} .
$$

The improper integral

$$
A_{f}=\frac{1}{2 \pi} \int_{\mathbb{R}^{2}} d \alpha d \beta \frac{\alpha^{2}+\beta^{2}}{2}|\alpha, \beta\rangle\langle\alpha, \beta|
$$

can be defined as the limit

$$
A_{f}=\lim _{d \rightarrow \infty} \int_{S_{d}} d \alpha d \beta \frac{\alpha^{2}+\beta^{2}}{2}|\alpha, \beta\rangle\langle\alpha, \beta|
$$

where $S_{d}$ is the square

$$
S_{d}=\left[-\left(s+\frac{1}{2}\right) \sqrt{\delta},\left(s+\frac{1}{2}\right) \sqrt{\delta}\right] \times\left[-\left(s+\frac{1}{2}\right) \sqrt{\delta},\left(s+\frac{1}{2}\right) \sqrt{\delta}\right] .
$$

By using a subdivison of $S_{d}$ into $d^{2}$ squares of side $\sqrt{\delta}=\sqrt{2 \pi / d}$, we can write $A_{f}$ as a limit of Riemann sums

$$
\begin{aligned}
A_{f} & =\lim _{d \rightarrow \infty} \frac{1}{2 \pi} \sum_{(\alpha, \beta) \in \mathcal{R}_{d}^{2}} \frac{2 \pi}{d} \frac{\alpha^{2}+\beta^{2}}{2}|\alpha, \beta\rangle\langle\alpha, \beta| \\
& =\lim _{d \rightarrow \infty} \frac{1}{d} \sum_{(\alpha, \beta) \in \mathcal{R}_{d}^{2}} \frac{\alpha^{2}+\beta^{2}}{2}|\alpha, \beta\rangle\langle\alpha, \beta| .
\end{aligned}
$$

For any $\psi$ we have

$$
\begin{aligned}
|\alpha, \beta\rangle\langle\alpha, \beta \mid \psi\rangle & =|\alpha, \beta\rangle \int_{\mathbb{R}} \overline{\mathcal{D}(\alpha, \beta) \Psi_{0}(x)} \psi(x) d x \\
& =\lim _{d \rightarrow \infty}|\alpha, \beta\rangle \int_{-\left(s+\frac{1}{2}\right) \sqrt{\delta}}^{\left(s+\frac{1}{2}\right) \sqrt{\delta}} \overline{\mathcal{D}(\alpha, \beta) \Psi_{0}(x)} \psi(x) d x .
\end{aligned}
$$


By using a subdivision of the interval $\left[-\left(s+\frac{1}{2}\right) \sqrt{\delta},\left(s+\frac{1}{2}\right) \sqrt{\delta}\right]$ into $d$ intervals of length $\sqrt{\delta}$ and the relation (155) we get

$$
\begin{aligned}
|\alpha, \beta\rangle\langle\alpha, \beta \mid \psi\rangle & =\lim _{d \rightarrow \infty}|\alpha, \beta\rangle \sum_{u \in \mathcal{R}_{d}} \sqrt{\delta} \overline{\mathcal{D}(\alpha, \beta) \Psi_{0}(u)} \psi(u) \\
& \approx \lim _{d \rightarrow \infty} \sqrt[4]{\delta}|\alpha, \beta\rangle \sum_{u \in \mathcal{R}_{d}} \overline{\mathbf{D}(\alpha, \beta) \mathbf{g}(u)} \psi(u) \\
& =\lim _{d \rightarrow \infty}|\alpha, \beta\rangle_{d d}\langle\alpha, \beta \mid \psi\rangle .
\end{aligned}
$$

Therefore, in a certain sense, we have

$$
A_{f}=\lim _{d \rightarrow \infty} \frac{1}{d} \sum_{(\alpha, \beta) \in \mathcal{R}_{d}^{2}} \frac{\alpha^{2}+\beta^{2}}{2}|\alpha, \beta\rangle_{d d}\langle\alpha, \beta| .
$$

From the relation

$$
\left\langle\varphi\left|\mathbf{A}_{\mathbf{f}}\right| \varphi\right\rangle=\left.\left.\frac{1}{d} \sum_{(\alpha, \beta) \in \mathcal{R}_{d}^{2}} \frac{\alpha^{2}+\beta^{2}}{2}\right|_{d}\langle\alpha, \beta \mid \varphi\rangle\right|^{2} \geq 0 \quad \text { for } \quad \varphi \in l^{2}\left(\mathcal{R}_{d}\right)
$$

it follows that the eigenvalues of $\mathbf{A}_{\mathbf{f}}$ are non-negative. The elements of the matrix $\left(\left\langle\varepsilon_{n}\left|\mathbf{H}_{d}\right| \varepsilon_{m}\right\rangle\right)_{-s \leq n, m \leq s}$ of $\mathbf{H}_{\mathbf{d}}$ in the basis $\left\{\varepsilon_{n}\right\}_{n=-s}^{s}$, namely,

$$
\left\langle\varepsilon_{n}\left|\mathbf{H}_{d}\right| \varepsilon_{m}\right\rangle=-\frac{1}{2} \delta_{n m}+\frac{\pi}{d^{2}} \sum_{a, b=-s}^{s}\left(a^{2}+b^{2}\right) \mathrm{e}^{\frac{2 \pi \mathrm{i}}{d} b(n-m)} \mathbf{g}((n-a) \sqrt{\delta}) \mathbf{g}((m-a) \sqrt{\delta})
$$

are real numbers described by periodic functions with respect to $n$ and $m$ and such that

$$
\left\langle\varepsilon_{n}\left|\mathbf{H}_{d}\right| \varepsilon_{m}\right\rangle=\left\langle\varepsilon_{m}\left|\mathbf{H}_{d}\right| \varepsilon_{n}\right\rangle=\left\langle\varepsilon_{-n}\left|\mathbf{H}_{d}\right| \varepsilon_{-m}\right\rangle .
$$

If $\left(v_{-s}, v_{-s+1}, \ldots, v_{s-1}, v_{s}\right)$ is an eigenvector of $\mathbf{H}_{d}$ corresponding to the eigenvalue $\lambda$ then

$$
\sum_{m=-s}^{s}\left\langle\varepsilon_{n}\left|\mathbf{H}_{d}\right| \varepsilon_{m}\right\rangle v_{m}=\lambda v_{n} \quad \text { for any } n \in\{-s,-s+1, \ldots, s-1, s\} .
$$

This relation being equivalent to

$$
\sum_{m=-s}^{s}\left\langle\varepsilon_{n}\left|\mathbf{H}_{d}\right| \varepsilon_{m}\right\rangle \bar{v}_{m}=\lambda \bar{v}_{n} \quad \text { for any } \quad n \in\{-s,-s+1, \ldots, s-1, s\}
$$

we can choose a real eigenvector. But, by using (79) the relation (80) can be written as

$$
\sum_{m=-s}^{s}\left\langle\varepsilon_{n}\left|\mathbf{H}_{d}\right| \varepsilon_{m}\right\rangle v_{-m}=\lambda v_{-n} \quad \text { for any } n \in\{-s,-s+1, \ldots, s-1, s\} .
$$

and this is possible only in the cases

$$
\left(v_{s}, v_{s-1}, \ldots, v_{-s+1}, v_{-s}\right)= \pm\left(v_{-s}, v_{-s+1}, \ldots, v_{s-1}, v_{s}\right) .
$$

Therefore any eigenfunction of $\mathbf{H}_{d}$ is either an even function or an odd one. By using some results concerning the centrosymmetric matrices [13], one can prove that $\mathbf{H}_{d}$ admits $s$ even and $s+1$ odd eigenfunctions.

The relation (42) and Theorem 2 allows us to write the diagonal elements as

$$
\left\langle\varepsilon_{n}\left|\mathbf{H}_{d}\right| \varepsilon_{n}\right\rangle=-\frac{1}{2}+\frac{\pi}{d} \frac{s(s+1)}{3}+\frac{\pi}{d} \sum_{a=-s}^{s} a^{2} \mathbf{g}^{2}((n-a) \sqrt{\delta})
$$


and the non-diagonal elements as

$$
\left\langle\varepsilon_{n}\left|\mathbf{H}_{d}\right| \varepsilon_{m}\right\rangle=\frac{1}{2} \mathbf{F}\left[\mathfrak{q}^{2}\right](n-m) \mathbf{F}\left[\mathbf{g}^{2}\right]((n-m) \sqrt{\delta}) .
$$

Particularly, we have

$$
\operatorname{tr} \mathbf{H}_{d}=-\frac{d}{2}+2 \pi \frac{s(s+1)}{3} .
$$

Numerically one can check (see Figure 3) that the eigenvalues of $\mathbf{H}_{d}$ are distinct and have the tendency to become $\frac{1}{2}, \frac{1}{2}+1, \frac{1}{2}+2, \ldots, \frac{1}{2}+d-1$, for large $d$. Since

$$
\sum_{n=0}^{d-1}\left(\frac{1}{2}+n\right)=\frac{d^{2}}{2}
$$

one can remark that

$$
\lim _{d \rightarrow \infty} \frac{\operatorname{tr} \mathbf{H}_{d}}{\sum_{n=0}^{d-1}\left(\frac{1}{2}+n\right)}=\frac{\pi}{3} \approx 1.0472 .
$$

Theorem 3. The matrix $\left(\left\langle\varepsilon_{n}\left|\mathbf{A}_{\mathbf{f}}\right| \varepsilon_{m}\right\rangle\right)_{-s \leq n, m \leq s}$ of $\mathbf{A}_{\mathbf{f}}=\mathbf{H}_{d}+\frac{1}{2}$ in the basis $\left\{\varepsilon_{n}\right\}_{n=-s}^{s}$ is

$$
\mathbf{A}_{\mathbf{f}}=\left(\begin{array}{llllllllll}
\omega_{s} & \tau_{1} & \tau_{2} & \ldots & \tau_{s-1} & \tau_{s} & \tau_{s} & \ldots & \tau_{2} & \tau_{1} \\
\tau_{1} & \omega_{s-1} & \tau_{1} & \ldots & \tau_{s-2} & \tau_{s-1} & \tau_{s} & \ldots & \tau_{3} & \tau_{2} \\
\tau_{2} & \tau_{1} & \omega_{s-2} & \ldots & \tau_{s-3} & \tau_{s-2} & \tau_{s-1} & \ldots & \tau_{4} & \tau_{3} \\
\vdots & \vdots & \vdots & \ddots & \vdots & \vdots & \vdots & \vdots & \vdots & \vdots \\
\tau_{s-1} & \tau_{s-2} & \tau_{s-3} & \ldots & \omega_{1} & \tau_{1} & \tau_{2} & \ldots & \tau_{s} & \tau_{s} \\
\tau_{s} & \tau_{s-1} & \tau_{s-2} & \ldots & \tau_{1} & \omega_{0} & \tau_{1} & \ldots & \tau_{s-1} & \tau_{s} \\
\tau_{s} & \tau_{s} & \tau_{s-1} & \ldots & \tau_{2} & \tau_{1} & \omega_{1} & \ldots & \tau_{s-2} & \tau_{s-1} \\
\vdots & \vdots & \vdots & \vdots & \vdots & \vdots & \vdots & \ddots & \vdots & \vdots \\
\tau_{2} & \tau_{3} & \tau_{4} & \ldots & \tau_{s} & \tau_{s-1} & \tau_{s-2} & \ldots & \omega_{s-1} & \tau_{1} \\
\tau_{1} & \tau_{2} & \tau_{3} & \ldots & \tau_{s} & \tau_{s} & \tau_{s-1} & \ldots & \tau_{1} & \omega_{s}
\end{array}\right)
$$

where

$$
\tau_{k}=\frac{1}{2 \sqrt{d}} \mathbf{F}\left[\mathfrak{q}^{2} * \mathbf{g}^{2}\right](k \sqrt{\delta}) \quad \text { and } \quad \omega_{k}=\tau_{0}+\frac{1}{2}\left(\mathfrak{q}^{2} * \mathbf{g}^{2}\right)(k \sqrt{\delta}) .
$$

Proof. The diagonal elements depend only on $|n|$ and the non-diagonal elements only on $|n-m|$ (see Figure 44). The relations (87) follow from (81), (82) and

$$
\tau_{0}=\frac{1}{2 d} \sum_{n=-s}^{s}\left(\mathfrak{q}^{2} * \mathbf{g}^{2}\right)(n \sqrt{\delta})=\frac{\pi}{d^{2}} \sum_{n, k=-s}^{s} k^{2} \mathbf{g}^{2}((n-k) \sqrt{\delta})=\frac{\pi}{d^{2}} \sum_{k=-s}^{s} k^{2}=\frac{\pi}{d} \frac{s(s+1)}{3} .
$$

The circulant matrix

$$
T_{d}=\left(\tau_{n-m}\right)_{-s \leq n, m \leq s}=\left(\begin{array}{llllll}
\tau_{0} & \tau_{1} & \tau_{2} & \ldots & \tau_{2} & \tau_{1} \\
\tau_{1} & \tau_{0} & \tau_{1} & \ldots & \tau_{3} & \tau_{2} \\
\tau_{2} & \tau_{1} & \tau_{0} & \ldots & \tau_{4} & \tau_{3} \\
\vdots & \vdots & \vdots & \ddots & \vdots & \vdots \\
\tau_{2} & \tau_{3} & \tau_{4} & \ldots & \tau_{0} & \tau_{1} \\
\tau_{1} & \tau_{2} & \tau_{3} & \ldots & \tau_{1} & \tau_{0}
\end{array}\right)
$$



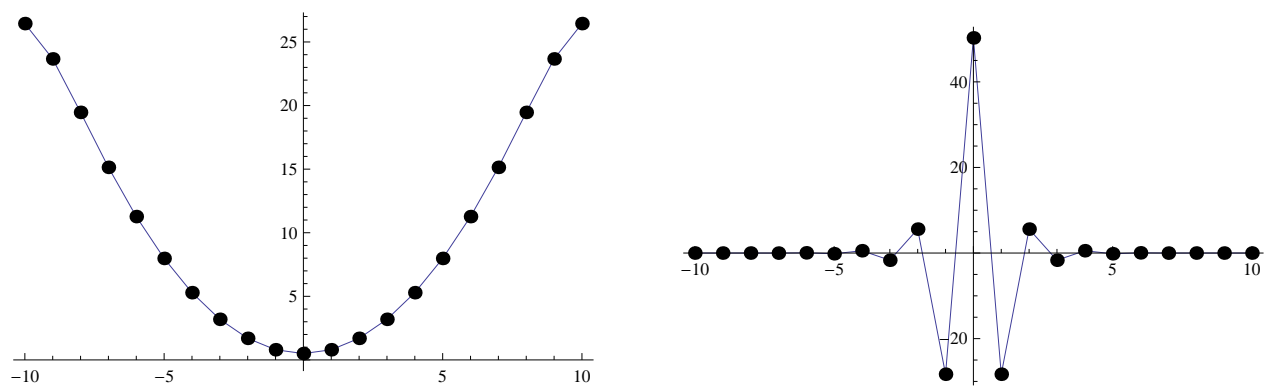

Figure 4. The convolution $\mathfrak{q}^{2} * \mathbf{g}^{2}$ (left) and its Fourier transform $\mathbf{F}\left[\mathfrak{q}^{2} * \mathbf{g}^{2}\right]$ (right).

has the eigenvalues 8]

$$
\sum_{n=-s}^{s} \tau_{n} \mathrm{e}^{\frac{2 \pi \mathrm{i}}{d} k n}=\frac{1}{2}\left(\mathfrak{q}^{2} * \mathbf{g}^{2}\right)(k \sqrt{\delta}) \quad \text { with } \quad k \in\{-s,-s+1, \ldots, s-1, s\}
$$

and satisfies the relation

$$
T_{d}=\mathbf{F}^{+} \mathfrak{D}_{f} \mathbf{F}
$$

where $\mathfrak{D}_{f}$ is the diagonal matrix

$$
\mathfrak{D}_{f}=\operatorname{diag}\left(\frac{1}{2}\left(\mathfrak{q}^{2} * \mathbf{g}^{2}\right)(-s \sqrt{\delta}), \frac{1}{2}\left(\mathfrak{q}^{2} * \mathbf{g}^{2}\right)((-s+1) \sqrt{\delta}), \ldots, \frac{1}{2}\left(\mathfrak{q}^{2} * \mathbf{g}^{2}\right)(s \sqrt{\delta})\right) .
$$

The matrix of $\mathbf{H}_{d}$ in the basis $\left\{\varepsilon_{n}\right\}_{n=-s}^{s}$ can be written as

$$
\mathbf{H}_{d}=-\frac{1}{2}+\mathfrak{D}_{f}+\mathbf{F}^{+} \mathfrak{D}_{f} \mathbf{F} .
$$

Up to a translation, it is similar to the matrix of $\frac{1}{2}\left(\mathbf{P}^{2}+\mathbf{Q}^{2}\right)$ which can be written as

$$
\frac{1}{2}\left(\mathbf{P}^{2}+\mathbf{Q}^{2}\right)=\mathfrak{D}+\mathbf{F}^{+} \mathfrak{D F} .
$$

by using the diagonal matrix

$$
\mathfrak{D}=\operatorname{diag}\left(\frac{1}{2} \mathfrak{q}^{2}(-s \sqrt{\delta}), \frac{1}{2} \mathfrak{q}^{2}((-s+1) \sqrt{\delta}), \ldots, \frac{1}{2} \mathfrak{q}^{2}(s \sqrt{\delta})\right) .
$$

Since $\mathbf{F}|\alpha, \beta\rangle_{d}=|-\beta, \alpha\rangle_{d}$, the mean value of $\mathbf{H}_{d}$ in the state $|\alpha, \beta\rangle_{d}$ is

$$
{ }_{d}\left\langle\alpha, \beta\left|\mathbf{H}_{d}\right| \alpha, \beta\right\rangle_{d}=-\frac{1}{2}+\frac{1}{2} \sum_{u \in \mathcal{R}_{d}}\left(\mathfrak{q}^{2} * \mathbf{g}^{2}\right)(u)\left(\mathbf{g}^{2}(u-\alpha)+\mathbf{g}^{2}(u-\beta)\right) .
$$

The circulant matrix with the equidistant eigenvalues $1,2,3, \ldots, d$ is

$$
C_{d}=\mathbf{F}^{+} \operatorname{diag}(1,2, \ldots, d) \mathbf{F}=\left(c_{n-m}\right)_{-s \leq n, m \leq s}
$$

where

$$
c_{k}=\frac{1}{d} \sum_{n=-s}^{s} n \mathrm{e}^{\frac{2 \pi \mathrm{i}}{d} k n}=\left\{\begin{array}{cc}
\frac{d+1}{2} & \text { for } k \in d \mathbb{Z} \\
\frac{\mathrm{e}^{\frac{2 \pi \mathrm{i}}{d} k}}{\mathrm{e}^{\frac{2 \pi \mathrm{i}}{d} k}-1} & \text { for } k \notin d \mathbb{Z} .
\end{array}\right.
$$

Wielandt-Hoffman theorem [8] admits as a direct consequence the following result. 
Theorem 4. 8] Given two Hermitian matrices $A=\left(a_{i j}\right)_{1 \leq i, j \leq d}$ and $B=\left(b_{i j}\right)_{1 \leq i, j \leq d}$ with eigenvalues $\alpha_{n}$ and $\beta_{n}$ in nondecreasing order, respectively, then

$$
\frac{1}{d} \sum_{k=1}^{d}\left|\alpha_{k}-\beta_{k}\right| \leq\|A-B\| \text {. }
$$

In view of this theorem, the eigenvalues $\lambda_{1}, \lambda_{2}, \ldots, \lambda_{d}$ of $\mathbf{A}_{\mathbf{f}}$ considered in a nondecreasing order satisfy the relation

$$
\frac{1}{d} \sum_{n=1}^{d}\left|n-\lambda_{n}\right| \leq\left(\sum_{k=1}^{d-1}\left|\tau_{k}-c_{k}\right|^{2}+\frac{1}{d} \sum_{k=-s}^{s}\left|\omega_{k}-c_{0}\right|^{2}\right)^{\frac{1}{2}} .
$$

Numerically one can check (see Figure 3) that $\lambda_{1}, \lambda_{2}, \ldots, \lambda_{d}$ have the tendency to become $1,2, \ldots, d$, for large $d$.

Theorem 5. The Hamiltonian $\mathbf{H}_{d}$ is Fourier invariant

$$
\mathbf{F H}_{d}=\mathbf{H}_{d} \mathbf{F} \text {. }
$$

Proof. By using the relation (56) we get

$$
\begin{aligned}
\mathbf{F H}_{d} \mathbf{F}^{+} & =-\frac{1}{2}+\frac{1}{d} \sum_{(\alpha, \beta) \in \mathcal{R}_{d}^{2}} \frac{\alpha^{2}+\beta^{2}}{2} \mathbf{F}|\alpha, \beta\rangle_{d d}\langle\alpha, \beta| \mathbf{F}^{+} \\
& =-\frac{1}{2}+\frac{1}{d} \sum_{(\alpha, \beta) \in \mathcal{R}_{d}^{2}} \frac{\alpha^{2}+\beta^{2}}{2}|-\beta, \alpha\rangle_{d d}\langle-\beta, \alpha|=\mathbf{H}_{d} .
\end{aligned}
$$

One can check that the normalized eigenfunctions $\mathbf{f}_{0}, \mathbf{f}_{1}, \ldots, \mathbf{f}_{d-1}$ of $\mathbf{H}_{d}$, considered in the increasing order of the number of sign alternations, satisfy the relation

$$
\mathbf{F} \mathbf{f}_{m}=(-\mathrm{i})^{m} \mathbf{f}_{m}
$$

and approximate the Hermite-Gaussian functions $\Psi_{m}$ better than the Harper functions $\mathbf{h}_{m}$. By denoting

$$
\begin{aligned}
& \Delta(m)=\max _{u \in \mathcal{R}_{d}}\left|\mathbf{f}_{m}(u)-\sqrt[4]{\delta} \Psi_{m}(u)\right| \\
& \Delta_{H}(m)=\max _{u \in \mathcal{R}_{d}}\left|\mathbf{h}_{m}(u)-\sqrt[4]{\delta} \Psi_{m}(u)\right|
\end{aligned}
$$

we have (see Figure 5 )

$$
\Delta(m)<\Delta_{H}(m) \quad \text { for almost all } m \in\{0,1,2, \ldots, d-1\} .
$$

\section{A finite counterpart of the raising operator}

The operator corresponding through the coherent state quantization to the function

$$
\mathbb{R}^{2} \longrightarrow \mathbb{C}:(\alpha, \beta) \mapsto \frac{\alpha-i \beta}{\sqrt{2}}
$$

is the usual raising operator [7]

$$
\frac{1}{2 \pi} \int_{\mathbb{R}^{2}} d \alpha d \beta \frac{\alpha-\mathrm{i} \beta}{\sqrt{2}}|\alpha, \beta\rangle\langle\alpha, \beta|=a^{+}
$$

satisfying the relations

$$
a^{+} \Psi_{n}=\sqrt{n+1} \Psi_{n+1} \quad \text { and } \quad \Psi_{n}=\frac{1}{\sqrt{n !}}\left(a^{+}\right)^{n} \Psi_{0}
$$




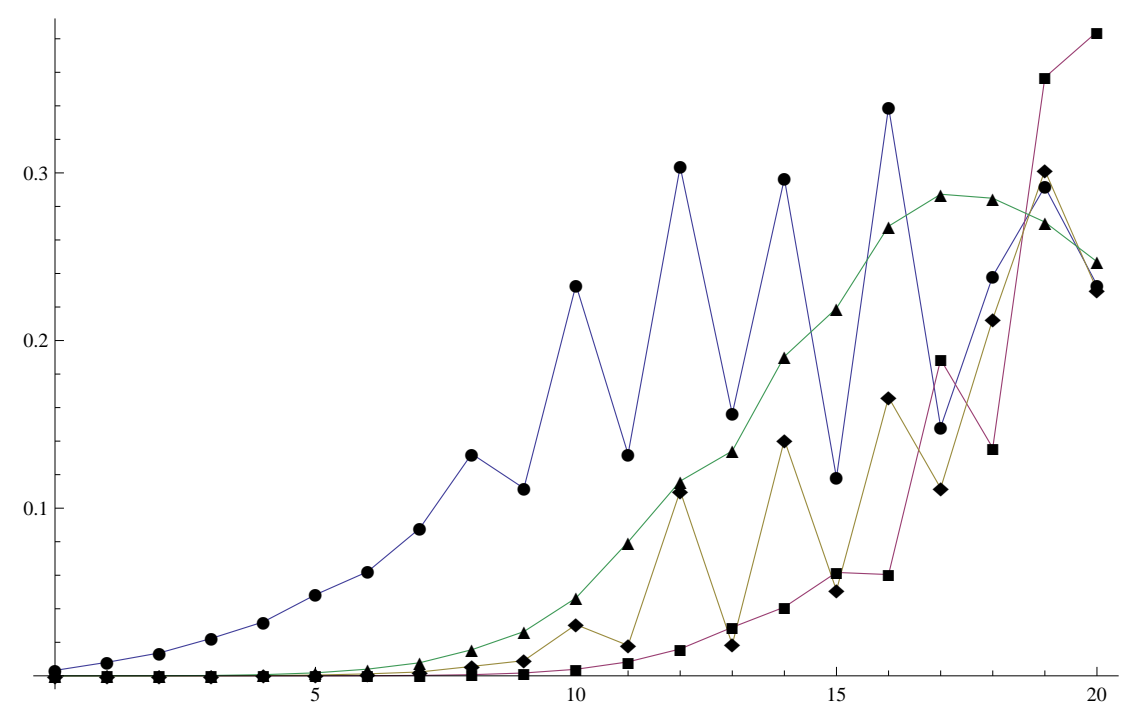

Figure 5. The values of $\Delta(m)$ (rhombs), $\Delta_{H}(m)$ (bullets), $\Delta_{M}(m)$ (squares) and $\Delta_{R}(m)$ (triangles) in the case $d=21$.

By using the finite frame quantization we define a finite counterpart of $a^{+}$, namely,

$$
\mathbf{a}_{d}^{+}: l^{2}\left(\mathcal{R}_{d}\right) \longrightarrow l^{2}\left(\mathcal{R}_{d}\right), \quad \mathbf{a}_{d}^{+}=\frac{1}{d} \sum_{(\alpha, \beta) \in \mathcal{R}_{d}^{2}} \frac{\alpha-i \beta}{\sqrt{2}}|\alpha, \beta\rangle_{d d}\langle\alpha, \beta|
$$

The elements of the matrix $\left(\left\langle\varepsilon_{n}\left|\mathbf{a}_{d}^{+}\right| \varepsilon_{m}\right\rangle\right)_{-s \leq n, m \leq s}$ of $\mathbf{a}_{d}^{+}$in the basis $\left\{\varepsilon_{n}\right\}_{n=-s}^{s}$, namely,

$$
\left\langle\varepsilon_{n}\left|\mathbf{a}_{d}^{+}\right| \varepsilon_{m}\right\rangle=\frac{1}{d} \sqrt{\frac{\pi}{d}} \sum_{a, b=-s}^{s}(a-\mathrm{i} b) \mathrm{e}^{\frac{2 \pi \mathrm{i}}{d} b(n-m)} \mathbf{g}((n-a) \sqrt{\delta}) \mathbf{g}((m-a) \sqrt{\delta})
$$

are real numbers described by periodic functions with respect to $n$ and $m$ and such that

$$
\left\langle\varepsilon_{n}\left|\mathbf{a}_{d}^{+}\right| \varepsilon_{m}\right\rangle=-\left\langle\varepsilon_{-n}\left|\mathbf{a}_{d}^{+}\right| \varepsilon_{-m}\right\rangle .
$$

The functions $\tilde{\mathbf{f}}_{0}, \tilde{\mathbf{f}}_{1}, \ldots, \tilde{\mathbf{f}}_{d-1}$ defined by the relation

$$
\tilde{\mathbf{f}}_{n}=\frac{1}{\sqrt{n !}}\left(\mathbf{a}_{d}^{+}\right)^{n} \mathbf{g}
$$

can be regarded as a finite counterpart of the Hermite-Gaussian functions. They satisfy the recurrence relation

$$
\mathbf{a}_{d}^{+} \tilde{\mathbf{f}}_{n}=\sqrt{n+1} \tilde{\mathbf{f}}_{n+1} .
$$

\section{A discrete fractional Fourier transform}

The continuous Fourier transform satisfies the relation

$$
\begin{aligned}
\mathcal{F}[\psi](x) & =\mathcal{F}\left[\sum_{m=0}^{\infty}\left\langle\Psi_{m}, \psi\right\rangle \Psi_{m}\right](x)=\sum_{m=0}^{\infty}\left\langle\Psi_{m}, \psi\right\rangle \mathcal{F}\left[\Psi_{m}\right](x) \\
& =\sum_{m=0}^{\infty}(-\mathrm{i})^{m} \Psi_{m}(x) \int_{-\infty}^{\infty} \Psi_{m}\left(x^{\prime}\right) \psi\left(x^{\prime}\right) d x^{\prime} \\
& =\int_{-\infty}^{\infty}\left[\sum_{m=0}^{\infty} \mathrm{e}^{-\frac{\pi \mathrm{i}}{2} m} \Psi_{m}(x) \Psi_{m}\left(x^{\prime}\right)\right] \psi\left(x^{\prime}\right) d x^{\prime}
\end{aligned}
$$


and the usual $\alpha$ th-order continuous fractional Fourier transform is defined as

$$
\mathcal{F}^{\alpha}[\psi](x)=\int_{-\infty}^{\infty}\left[\sum_{m=0}^{\infty} \mathrm{e}^{-\frac{\pi \mathrm{i}}{2} m \alpha} \Psi_{m}(x) \Psi_{m}\left(x^{\prime}\right)\right] \psi\left(x^{\prime}\right) d x^{\prime} .
$$

The finite Fourier transform $l^{2}\left(\mathcal{R}_{d}\right) \longrightarrow l^{2}\left(\mathcal{R}_{d}\right): \varphi \mapsto \mathbf{F}[\varphi]$ satisfies the relation

$$
\begin{aligned}
\mathbf{F}[\varphi](u) & =\mathbf{F}\left[\sum_{m=0}^{d-1}\left\langle\mathbf{h}_{m}, \varphi\right\rangle \mathbf{h}_{m}\right](u)=\sum_{m=0}^{d-1}\left\langle\mathbf{h}_{m}, \varphi\right\rangle \mathbf{F}\left[\mathbf{h}_{m}\right](u) \\
& =\sum_{m=0}^{d-1}(-\mathrm{i})^{m} \mathbf{h}_{m}(x) \sum_{v \in \mathcal{R}_{d}} \mathbf{h}_{m}(v) \varphi(v) \\
& =\sum_{v \in \mathcal{R}_{d}}\left[\sum_{m=0}^{d-1} \mathrm{e}^{-\frac{\pi \mathrm{i}}{2} m} \mathbf{h}_{m}(u) \mathbf{h}_{m}(v)\right] \varphi(v) .
\end{aligned}
$$

and the transformation

$$
l^{2}\left(\mathcal{R}_{d}\right) \longrightarrow l^{2}\left(\mathcal{R}_{d}\right): \varphi \mapsto \mathbf{F}_{H}^{\alpha}[\varphi]
$$

where

$$
\mathbf{F}_{H}^{\alpha}[\varphi](u)=\sum_{v \in \mathcal{R}_{d}}\left[\sum_{m=0}^{d-1} \mathrm{e}^{-\frac{\pi \mathrm{i}}{2} m \alpha} \mathbf{h}_{m}(u) \mathbf{h}_{m}(v)\right] \varphi(v)
$$

is called a discrete fractional Fourier transform. It is a unitary transformation

$$
\mathbf{F}_{H}^{\alpha}\left(\mathbf{F}_{H}^{\alpha}\right)^{+}=\left(\mathbf{F}_{H}^{\alpha}\right)^{+} \mathbf{F}_{H}^{\alpha}=\mathbf{I}
$$

and satisfies the relations

$$
\mathbf{F}_{H}^{0}=\mathbf{I}, \quad \mathbf{F}_{H}^{1}=\mathbf{F} \quad \text { and } \quad \mathbf{F}_{H}^{\alpha} \mathbf{F}_{H}^{\beta}=\mathbf{F}_{H}^{\alpha+\beta} .
$$

\section{An alternative discrete fractional Fourier transform}

The finite Fourier transform $l^{2}\left(\mathcal{R}_{d}\right) \longrightarrow l^{2}\left(\mathcal{R}_{d}\right): \varphi \mapsto \mathbf{F}[\varphi]$ satisfies the relation

$$
\mathbf{F}[\varphi](u)=\sum_{v \in \mathcal{R}_{d}}\left[\sum_{m=0}^{d-1} \mathrm{e}^{-\frac{\pi \mathrm{i}}{2} m} \mathbf{f}_{m}(u) \mathbf{f}_{m}(v)\right] \varphi(v)
$$

and the transformation

$$
l^{2}\left(\mathcal{R}_{d}\right) \longrightarrow l^{2}\left(\mathcal{R}_{d}\right): \varphi \mapsto \mathbf{F}^{\alpha}[\varphi]
$$

where

$$
\mathbf{F}^{\alpha}[\varphi](u)=\sum_{v \in \mathcal{R}_{d}}\left[\sum_{m=0}^{d-1} \mathrm{e}^{-\frac{\pi \mathrm{i}}{2} m \alpha} \mathbf{f}_{m}(u) \mathbf{f}_{m}(v)\right] \varphi(v)
$$

is also a discrete fractional Fourier transform. It is a unitary transformation

$$
\mathbf{F}^{\alpha}\left(\mathbf{F}^{\alpha}\right)^{+}=\left(\mathbf{F}^{\alpha}\right)^{+} \mathbf{F}^{\alpha}=\mathbf{I}
$$

and satisfies the relations

$$
\mathbf{F}^{0}=\mathbf{I}, \quad \mathbf{F}^{1}=\mathbf{F} \quad \text { and } \quad \mathbf{F}^{\alpha} \mathbf{F}^{\beta}=\mathbf{F}^{\alpha+\beta} .
$$



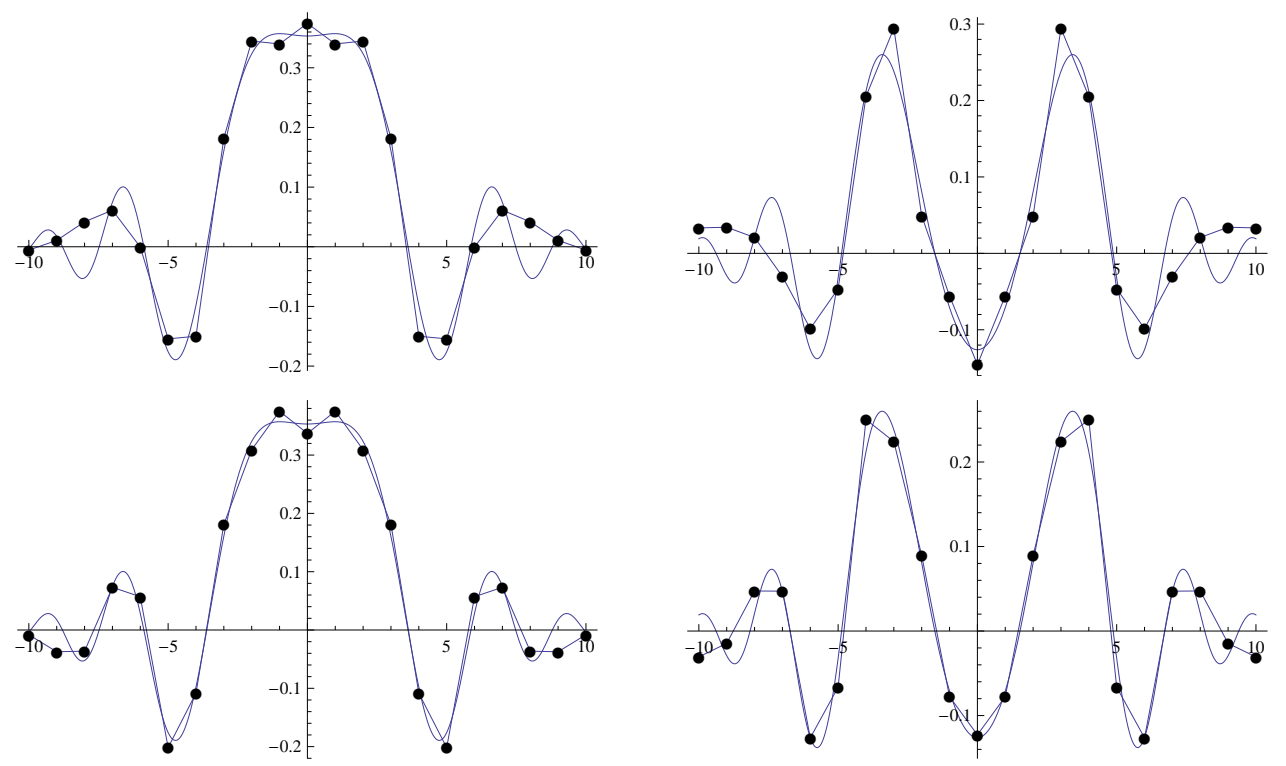

Figure 6. The real part (left hand side) and the imaginary part (right hand side) of the 0.5-th order fractional Fourier transform of the Gaussian $g_{10}$ and of its discrete counterpart $\mathfrak{g}_{10}$ computed by using the definition based on the Harper functions (first line) and the proposed definition (second line).

The discrete fractional Fourier transform computed by using our definition (119), generally, approximates the corresponding continuous fractional Fourier transform better than the discrete fractional transformation computed by using the definition (115) based on the Harper functions. For example, in the case $d=21$, the discrete counterpart of the Gaussian

$$
g_{10}: \mathbb{R} \longrightarrow \mathbb{R}, \quad g_{10}(x)=\mathrm{e}^{-5 x^{2}}
$$

is the periodic function (see Figure 1)

$$
\mathfrak{g}_{10}: \mathbb{Z} \sqrt{\delta} \longrightarrow \mathbb{R}, \quad \mathfrak{g}_{10}(n \sqrt{\delta})=\sum_{\ell=-\infty}^{\infty} \mathrm{e}^{-\frac{10 \pi}{21}(21 \ell+n)^{2}} .
$$

In Figure [ we present the graphs of the real and imaginary part of $\mathcal{F}^{\alpha}\left[g_{10}\right]$ superposed on the corresponding graphs of $\mathbf{F}_{H}^{\alpha}\left[\mathfrak{g}_{10}\right]$ (first line) and $\mathbf{F}^{\alpha}\left[\mathfrak{g}_{10}\right]$ (second line). In Figure 7 we compare the 0.5 -th order fractional Fourier transforms of the rectangular function

$$
\psi: \mathbb{R} \longrightarrow \mathbb{R}, \quad \psi(x)=\left\{\begin{array}{lll}
1 & \text { for } & x \in[-\sqrt{\delta}, \sqrt{\delta}] \\
0 & \text { for } & x \notin[-\sqrt{\delta}, \sqrt{\delta}]
\end{array}\right.
$$

and of its discrete counterpart

$$
\varphi: \mathcal{R}_{21} \longrightarrow \mathbb{R}, \quad \varphi(x)=\left\{\begin{array}{lll}
1 & \text { for } & x \in\{-\sqrt{\delta}, 0, \sqrt{\delta}\} \\
0 & \text { for } & x \notin\{-\sqrt{\delta}, 0, \sqrt{\delta}\}
\end{array}\right.
$$

computed by using the definition based on the Harper functions and our definition. 

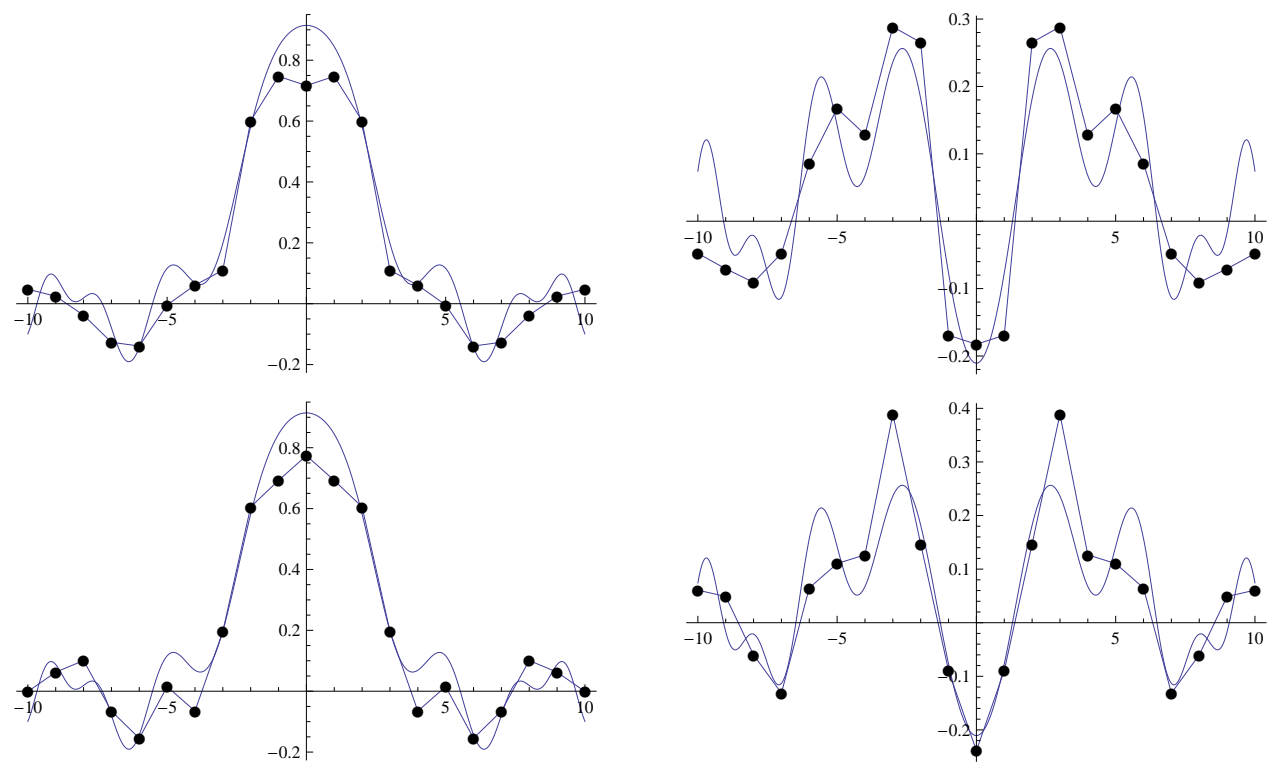

Figure 7. The real part (left hand side) and the imaginary part (right hand side) of the 0.5-th order fractional Fourier transform of the rectangular function (124) and of its discrete counterpart (125) computed by using the definition based on the Harper functions (first line) and the proposed definition (second line).

\section{Concluding remarks}

In the case of a particle moving along a line, the momentum operator admits the differential representation

$$
\hat{p}=-\mathrm{i} \frac{\mathrm{d}}{\mathrm{d} q}
$$

as well as the integral representation

$$
(\hat{p} \psi)(q)=\frac{1}{2 \pi} \int_{\mathbb{R}^{2}} x \mathrm{e}^{\mathrm{i} x y} \psi(q-y) d x d y
$$

equivalent to $\hat{p}=\mathcal{F}^{+} \hat{q} \mathcal{F}$. In the case of a quantum system with finite-dimensional Hilbert space, the definition of the momentum operator is obtained by starting from the integral representation, and not as finite difference operator.

The Hamiltonian of the harmonic oscillator admits the differential representation

$$
\hat{H}=-\frac{1}{2} \frac{\mathrm{d}^{2}}{\mathrm{~d} x^{2}}+\frac{1}{2} x^{2}
$$

as well as the integral representation

$$
\hat{H}=-\frac{1}{2}+\frac{1}{2 \pi} \int_{\mathbb{R}^{2}} d \alpha d \beta \frac{\alpha^{2}+\beta^{2}}{2}|\alpha, \beta\rangle\langle\alpha, \beta| .
$$

A finite counterpart is usually obtained by using a difference operator instead of the differential operator. We think that, generally, the integral representations behave better than the differential representations when we have to define finite versions. The finite frame quantization is a finite counterpart of the coherent state quantization, and we have used it in order to define a finite oscillator by starting from the integral representation 
(129). We belive that it approximates the harmonic oscillator better than the finite version based on the use of finite difference operators (see relation (85), Figure 3 and Figure 5).

There exists several systems of functions which can be regarded as a finite counterpart of the Hermite-Gauss functions. Among them there are the Mehta functions

$$
\Phi_{m}(n)=\sum_{\ell=-\infty}^{\infty} \Psi_{m}((\ell d+n) \sqrt{\delta})
$$

the Harper functions $\left\{\mathbf{h}_{m}\right\}$, and our systems of functions $\left\{\mathbf{f}_{m}\right\}$ and $\left\{\tilde{\mathbf{f}}_{m}\right\}$. In Figure 5 these systems are compared with the Hermite-Gauss functions by using the notations (102) and

$$
\begin{aligned}
& \Delta_{M}(m)=\max _{u \in \mathcal{R}_{d}}\left|\Phi_{m}(u)-\sqrt[4]{\delta} \Psi_{m}(u)\right| \\
& \Delta_{R}(m)=\max _{u \in \mathcal{R}_{d}}\left|\tilde{\mathbf{f}}_{m}(u)-\sqrt[4]{\delta} \Psi_{m}(u)\right| .
\end{aligned}
$$

\section{References}

[1] Barker L, Candan Ç, Hakioğlu T, Kutay M A and Ozaktas H M 2000 The discrete harmonic oscillator, Harper's equation, and the discrete fractional Fourier transform J. Phys. A: Math. Gen. 33 2209-22

[2] Candan Ç, Kutay M A and Ozaktas H M 2000 The discrete fractional Fourier transform IEEE Trans. Signal Process. 48, 1329

[3] Christensen O 2003 An introduction to frames and Riesz bases (Boston: Birkhäuser)

[4] Cotfas N and Dragoman D 2012 Properties of finite Gaussians and the discrete-continuous transition J. Phys. A: Math. Theor. 45425305

[5] Cotfas N and Gazeau J P 2010 Finite tight frames and some applications J. Phys. A: Math. Theor. 43193001

[6] Cotfas N, Gazeau J P and Vourdas A 2011 Finite-dimensional Hilbert space and frame quantization J. Phys. A: Math. Theor. 44175303

[7] Gazeau J-P 2009 Coherent States in Quantum Physics (Berlin: Wiley-VCH)

[8] Gray R M 2006 Toeplitz and Circulant Matrices: A review Foundations and Trends in Communications and Information Theory 2 155-239

[9] Han D and Larson D R 2000 Frames, bases and group representations Mem. Amer. Math. Soc. 697

[10] Marchiolli M A and Ruzzi M 2012 Theoretical formulation of finite-dimensional discrete phase spaces: I. Algebraic structures and uncertainty principles Ann. Phys. 327 1538-1461

[11] Marzoli I, Saif F, Bialynicki-Birula I, Friesch O M, Kaplan A E and Schleich W P 1998 Quantum carpets made simple Acta Phys. Slovaca 48323

[12] Mehta M L 1987 Eigenvalues and eigenvectors of the finite Fourier transform J. Math. Phys. 28 781

[13] Muthiyalu N and Usha S 1992 Eigenvalues of centrosymmetric matrices Computing 48213

[14] Ozaktas H M, Zalevsky Z and Kutay M A 2001 The Fractional Fourier Transform with Applications in Optics and Signal Processing (Chichester: John Wiley \& Sons )

[15] Perelomov A M 1986 Generalized Coherent States and their Applications (Berlin: Springer)

[16] Ruzzi M 2006 Jacobi $\theta$-functions and discrete Fourier transform J. Math. Phys. 47063507

[17] Santhanam T S and Tekumalla A R 1976 Quantum mechanics in finite dimensions Found. Phys. 6 583-589

[18] Schwinger J 1960 Unitary operator bases Proc. Natl. Acad. Sci. (USA) 46570 
[19] Štoviček P and Tolar J 1984 Quantum mechanics in discrete space-time Rep. Math. Phys. 20 157-70

[20] Tolar J and Chadzitaskos G 1997 Quantization on $\mathbb{Z}_{M}$ and coherent states over $\mathbb{Z}_{M} \times \mathbb{Z}_{M}$ J. Phys. A: Math. Gen. 30 2509-2517

[21] Vourdas A 2004 Quantum systems with finite Hilbert space Rep. Prog. Phys. 67 267-320

[22] Wolf K B and Krötzsch G 2007 Geometry and dynamics in the fractional discrete Fourier transform J. Opt. Soc. Am. A $2 \mathbf{4} 651-8$

[23] Zak J 1967 Finite translations in solid state physics Phys. Rev. Lett. 191385

[24] Zhang S and Vourdas A 2004 Analytic representation of finite quantum systems J. Phys. A: Math. Gen. 37 8349-63 